\title{
Adult Level of Physical Activity in Urban (Abobo, Cocody) and Rural (Yocoboué) Area in Côte d'Ivoire
}

\author{
Nina Laurette Ahouéfa ${ }^{1}$, Audrey Herbert Yepié ${ }^{1}$, Jean Jacques Diagou ${ }^{1}$ \& Louise Anin-Atchibri ${ }^{1}$ \\ ${ }^{1}$ Laboratory of Nutrition and Food Safety, UFR of Sciences and Food Technologies, Nangui Abrogoua \\ University, Abidjan, Côte d'Ivoire \\ Correspondence: Ahouéfa Nina Laurette, Laboratory of Nutrition and Food Safety, UFR of Sciences and Food \\ Technologies, Nangui Abrogoua University, Abidjan, 02 BP 801 Abidjan 02, Côte d'Ivoire. Tel: 225-0978-8910. \\ E-mail: laurette.nina2017@gmail.com
}

Received: August 13, 2019

doi:10.5539/jfr.v8n6p154
Accepted: October 29, 2019 Online Published: November 20, 2019

URL: https://doi.org/10.5539/jfr.v8n6p154

\begin{abstract}
The physical activity (PA) plays an important role in the fight against the chronic noncommunicable diseases.The objective of this study was to assess the level of physical activity in an adult population in rural and urban area in Côte d'Ivoire. To achieve this goal, a group of 1046 participants was submitted to the Global Physical Activity Questionnaire (GPAQ). The data analysis was done according to the GPAQ guidelines. The prevalence of the recommended level of the physical activity during the work, the leisure and the displacement was calculated.This assessment has showed that the intense Physical Activity (PA) decreased with age, from 34\% (19-29 years) to $14 \%$ (50-60 years).Then the results have showed that the workers had the highest intensity Physical Activity Level (NAP) at 70\%, versus 13\% for the office workers.Moreover, more the Level of Study (LS) was higher, more the physical activity practiced decreased, whatever the area of living. Finally, it has been shown that men were more active than women. Also, the level of physical activity was higher in rural than in urban areas, and the limited physical activity level was present in the adult population. This work has shown that the level of physical activity and the contexts of practice vary according to the socio-demographic characteristics. At that fact, targeted interventions to promote the physical activity are necessary.
\end{abstract}

Keywords: assessment, physical activity, Global Questionnaire on Physical Activity (GPAQ), rural, urban, adult, Côte d'Ivoire

\section{Introduction}

The inactivity is the fourth leading cause of death in the world and is now presented as a true pandemic (Kohl et al., 2012). In fact, the change in lifestyle, the sedentarization of professional activities, the motorization of the means of transport and the automation of housework, have been accompanied by a decrease in the energy expenditure of the population (HCSP, 2000; INSERM, 2008). However, the practice of a regular and adapted physical activity, as well as the limitation of the sedentary activities, constitute with the adoption of a balanced diet, the major factors of acquisition and maintenance of a good state of health. The physical activity is involved, at the same title than the eating habits, in the prevention of a large number of chronic diseases (cardiovascular diseases, diabetes, obesity, cancer, etc.) (INSERM, 2008). Thus, with the 2008 Health Nutrition Barometer, the level of physical activity and that of the sedentarity of the population were assessed using the World Physical Activity Questionnaire (GPAQ) of the World Health Organization (OMS, 2005). It permits to study the contexts of practice (work, leisure, displacement) and the levels of physical activity (high, medium, and limited) during a typical week. In this context, the promotion of the physical activity and the fight against the sedentarity are the major objectives, particularly in the fight against the noncommunicable diseases (OMS, 2005).

Several surveys conducted at the initiative of the health sector have focused on the physical activity and the sedentary practices of the French population (Barnett et al., 2008). However, in the countries of sub-Saharan Africa, a few data on the level of physical activity of the populations are available (Guthold et al., 2008). In Côte d'Ivoire, some studies have estimated the prevalence of the physical activity at $66.7 \%$ using the IPAQ method, which is recognized as overestimating the data (Guthold et al., 2008). Also the study conducted by Pepe (2017) has only highlighted the importance of the physical activity as a means of fighting against the noncommunicable diseases. Thereby, the objective of this study was to assess the level of physical activity according to the 
socio-demographic characteristics of a rural (Yocoboué) and urban (Abobo and Cocody) adult population in Côte d'Ivoire.

\section{Material and Methods}

\subsection{Study Area}

The study was conducted in Côte d'Ivoire, in two (2) communes of Abidjan (Abobo and Cocody), and in a village in the south-east of Côte d'Ivoire (Yocoboué).

\subsection{Type of Study, Target Population and Sampling}

A descriptive and analytical cross-sectional study was conducted from August 22 to November 19, 2018, and has involved adults. The study population was composed of one thousand one hundred and forty-six (1146) apparently healthy subjects (without declared diseases), aged from 18 to 60 years, including 688 women and 458 men, living in Abidjan (Abobo and Cocody) and in a south-east village of Côte d'Ivoire (Yocoboué).

The subjects were selected according to the simple random method (Giezendanner, 2012) in 6 areas of each urban (Abobo and Cocody) and rural (Yocoboué) region. We used the formula of Giezendanner (2012) for calculating the sample size. The pregnant and lactating women were excluded from the study. The study participants must have resided in the areas of selection for at least 6 months. They must have been previously informed of the purpose of the study and have given their consent of participation.

\subsection{Technics and Collection Tools}

The data collection technics used were the questionnaire survey for adults. The questionnaire inspired by the WHO STEPS instrument (WHO, 2005) collected information on the physical activity practice and the socio-demographic characteristics such as age, gender, work status, place of residence and the level of education.

\subsection{Measuring the Level of Physical Activity}

To assess the level of physical activity (PAN) in the study population, the Global Physical Activity Questionnaire (GPAQ) was used. The GPAQ questionnaire, developed by the WHO, contains 16 questions and collects information on the practice of physical activity during activities performed at work, at leisure or a displacement. The activities at work include paid and unpaid work and the housework. The leisure activities include sport and those related to displacement of at least 10 minutes on foot, by bike or in wheel skates. Each participant was asked to describe their weekly physical activity during his professional activities, displacements and leisure. The GPAQ analysis guide provided by WHO was used to analyze the data (Maceraet Pratt, 2000).

\subsection{Statistical Analysis}

The data analysis was performed using the IBM SPSS statistical analysis software version 20 (SPSS for windows, SPSS Inc., Chicago, IL). The results were presented under the form of percentage. The comparisons of proportions were made by the Pearson chi-square test at 0.05 significance level

\section{Results}

\subsection{Socio-demographic Characteristics of the Study Population}

The socio-demographic characteristics of the study population are presented in Table 1 . The average age of adults was $38.72 \pm 18.5$ years, ranging from 18 to 60 years. The modal class is between 30 and 39 years old (49\%).Of the 1146 participants, 688 (60.03\%) were female, 768 (67\%) come from urban areas of Abidjan (Abobo and Cocody) and 33\% from rural areas (Yocoboué).

$67.36 \%$ were educated and the most representative socio professional category of this study was the liberal activity with $47.73 \%$ of the participants. The category of civil servants was presented in two groups, namely clerical agents and workers. Workers were present only in urban areas (Abobo 8\% and Cocody 5\%). 
Table 1. Socio-demographic characteristics of the study population

\begin{tabular}{|c|c|c|c|c|c|c|}
\hline & Rural area & & Urban area & & & \\
\hline \multirow{2}{*}{ Total $(n=1146)$} & \multicolumn{2}{|c|}{ Yocoboué $(n=378)$} & \multicolumn{2}{|c|}{ Abobo $(n=384)$} & \multicolumn{2}{|c|}{ Cocody $(n=384)$} \\
\hline & $\mathbf{n}$ & $\%$ & $\mathbf{n}$ & $\%$ & $\mathbf{n}$ & $\%$ \\
\hline \multicolumn{7}{|l|}{ Sex } \\
\hline Men & 151 & & 163 & & 144 & \\
\hline Women & 227 & & 221 & & 240 & \\
\hline Total & 378 & & 384 & & 384 & \\
\hline Averageage & $39,76 \pm 17,9$ & & $37,77 \pm 18,69$ & & $38,63 \pm 18,92$ & \\
\hline \multicolumn{7}{|l|}{ Age range (year) } \\
\hline - $18-29$ & 137 & 36,3 & 146 & 38 & 116 & 30,2 \\
\hline - $\quad 30-39$ & 188 & 49,7 & 188 & 49 & 185 & 48,2 \\
\hline - $40-49$ & 30 & 8 & 40 & 10,4 & 51 & 13,3 \\
\hline - $\quad 50-60$ & 23 & 6 & 10 & 2,6 & 32 & 8,3 \\
\hline \multicolumn{7}{|l|}{ Education level } \\
\hline - Uneducated & 208 & 55 & 106 & 27,6 & 60 & 15,6 \\
\hline - Educated & 170 & 45 & 278 & 72,4 & 324 & 84,4 \\
\hline - Primairy & 106 & 28 & 95 & 24,7 & 50 & 13 \\
\hline - Secondairy & 60 & 16 & 102 & 26,5 & 146 & 38 \\
\hline - Superior & 4 & 1 & 81 & 21,1 & 128 & 33,3 \\
\hline \multicolumn{7}{|l|}{ Professional status } \\
\hline - Unemployed & 38 & 10 & 62 & 16 & 29 & 7,0 \\
\hline - Housewives & 54 & 14 & 46 & 12 & 8 & 2,0 \\
\hline - Oficials & 71 & 19 & 84 & 22 & 189 & 49,0 \\
\hline - Office Agent & 71 & 19 & 54 & 14 & 171 & 45,0 \\
\hline - Workers & - & - & 30 & 8 & 18 & 5,0 \\
\hline - Liberal activity & 215 & 57 & 192 & 50 & 140 & 36,0 \\
\hline
\end{tabular}

\subsection{Contexts of Practice according to the Physical Activity Level}

The distribution of the level of the physical activity according to the context of practice (high, moderate, limited) is shown in Figure 1. It shows that among the populations with a high level of physical activity, the work accounts for the most part of the total physical activity, with respectively $64.8 \%$ in Yocoboue against and $68.2 \%$ in Abobo and 24.7\% in Cocody. While the displacement-related physical activity is observed in moderate-level in Yocoboué populations at $48.2 \%$, in high-level in populations of Abobo at $48.2 \%$ and in limited-level in populations of Cocody at $40.4 \%$.

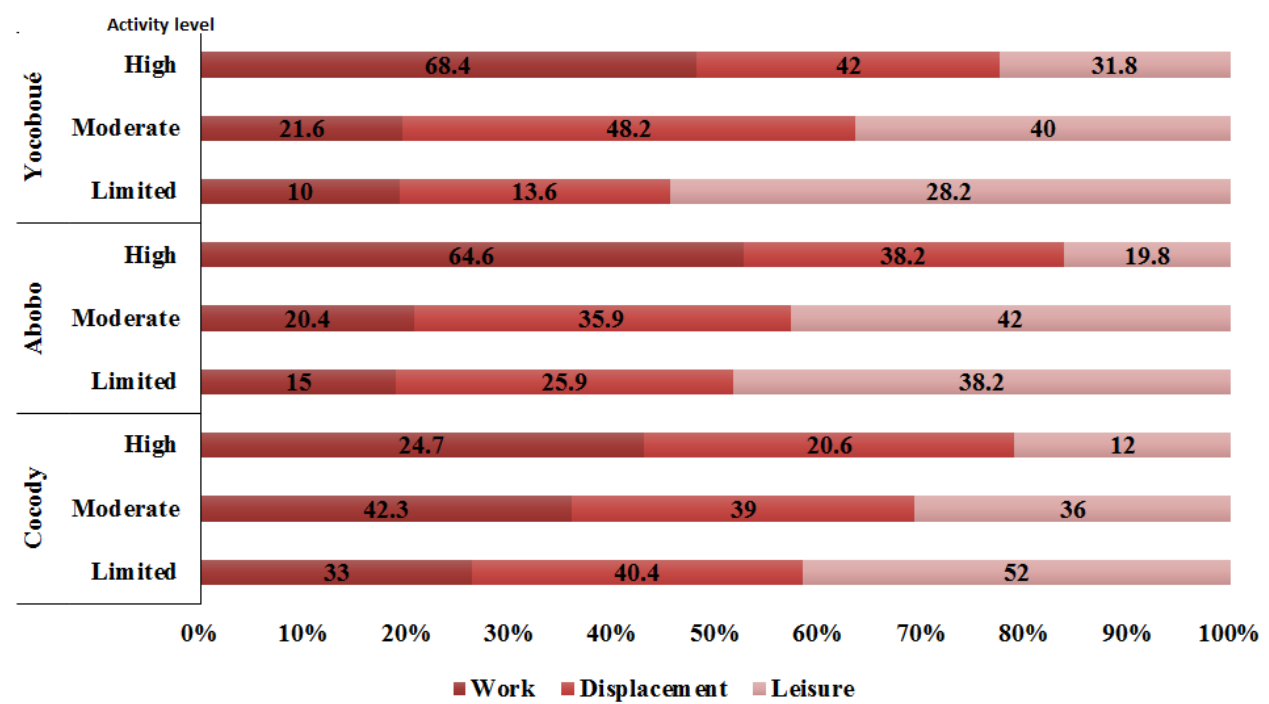

Figure 1. Contexts of practice according to the physical activity level 


\subsection{Level of "High" Physical Activity according to the Age}

The age distribution of the study population according to the physical activity level is shown in figure 2 . According to the categorization of the physical activity defined by the WHO from the GPAQ questionnaire, the level of intense physical activity decreased with age. Respectively $22.0 \%, 18.0 \%, 10.8 \%$ and $3.9 \%$ for the age groups [18-29years], [30-39 years], [40-49 years] and [50-60 years] among the inhabitants of Cocody. Then $35.0 \%, 20.0 \%, 19.5 \%$ and $8.0 \%$ for the age groups [18-29 years], [30-39 years], [40-49 years] and [50-60 years] among the inhabitants of Abobo. Finally, 45.2\%, 40.6\%, 27.2\%, and 21.9\% for the age groups [18-29 years], [30-39 years], [40-49years] and [50-60 years] for the populations of Yocoboué.

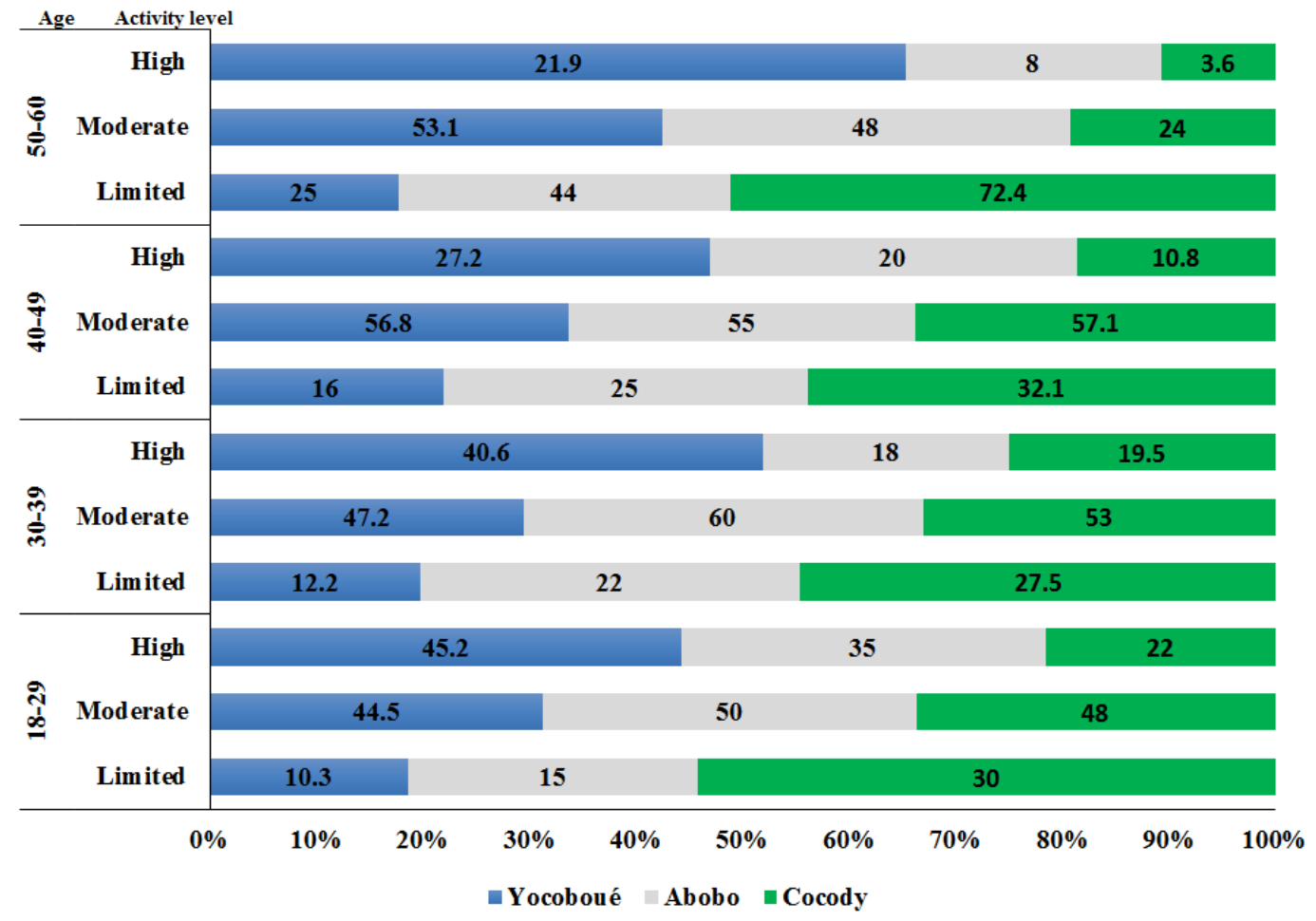

Figure 2. Age distribution of the study population according to the physical activity level

\subsection{Physical Activity Level According the Socio-professional Category}

The figure 3 shows the physical activity level (PAL) of the study participants according to their socio-professional category. From all the socio-professional categories of the entire sample, the workers were those who had the most intense PAL (70.8\%), followed by the liberal activities leaders (42.7\%), the housewives (36\%), the unemployed (28\%) and finally the office workers (13\%). The intense physical activity level (PAL) was higher among people practicing a liberal activity in Yocoboué (rural area), with $75 \%$ of prevalence, than in urban areas (Abobo 32.6\% and Cocody 7.1\%). The housewives of Yocoboué (rural area) had an intense Physical Activity more important $(59,7 \%)$ than those of urban area of Abobo $(13 \%)$ and Cocody $(8,5 \%)$. For the office agents, we noted that those of Cocody (79.4\%) and Abobo (29\%) had a limited physical activity level (PAL) more important than those of Yocoboue (18.29\%). The physical activity of the unemployed is mostly moderate in Yocoboué (48\%) and Abobo (37.5\%) and rather limited (60\%) in Cocody 


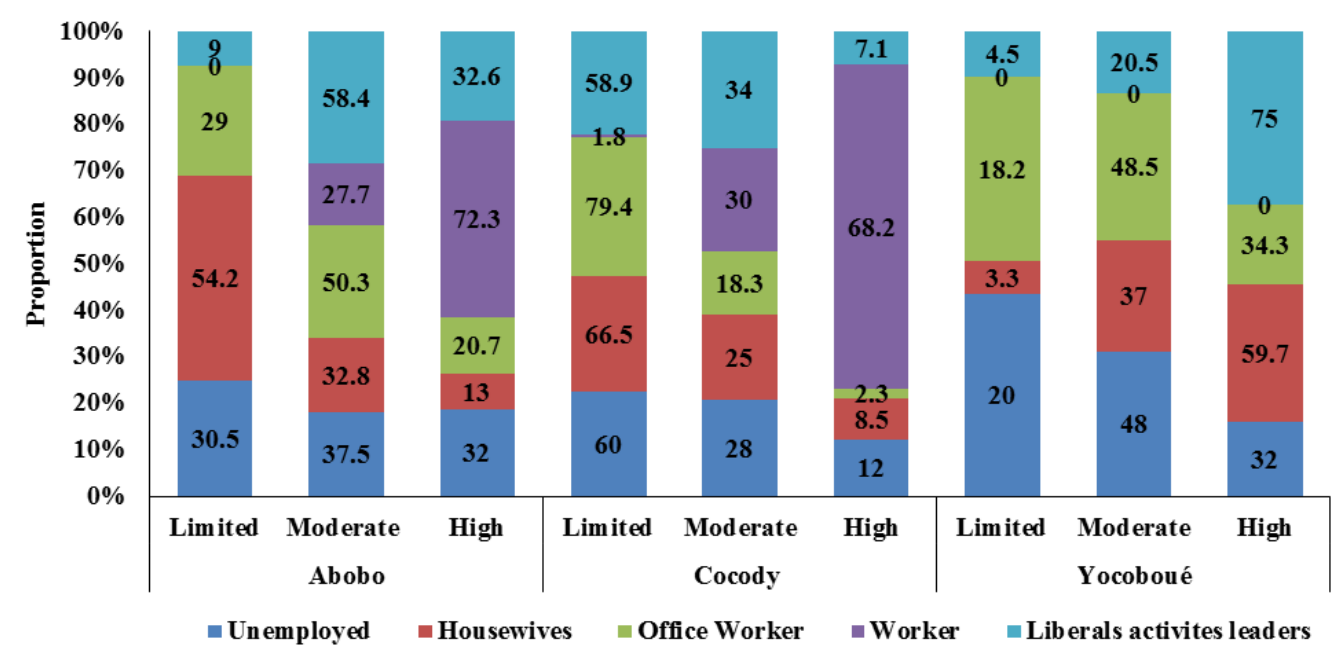

Figure 3. Distribution of the physical activity level (PAL) according to the socio-professional

\subsection{Level of "High" Physical Activity according to the Sex}

The level of physical activity according to the sex is shown in Figure 4. The intensive Physical activity in men is more important than in women, from rural areas to urban areas. However, it should be noted that the men of Yocoboué (rural) are more active than those of the urban areas of Abobo (50.6\%) and Cocody (10.6\%). The urban women are less active than those of the rural areas, and the men of the urban areas are much more physically active.

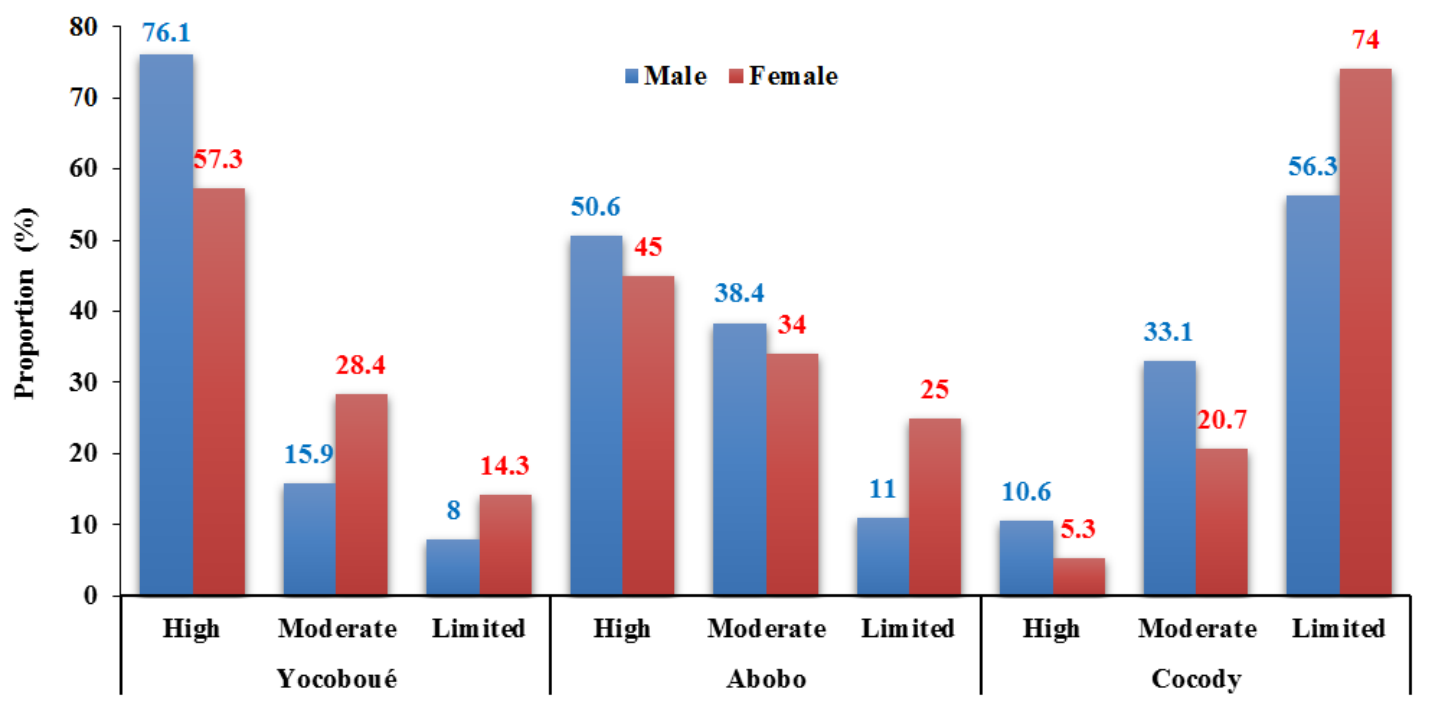

Figure 4. Distribution of the physical activity level (PAL) according to the sex

\subsection{Physical Activity according to Study Level}

The Figure 5 shows the distribution of the physical activity among the study participants according to the study level. The physical activity of the participants with no education was more important in allthe areas of residence, but was much more pronounced in rural areas (Yocoboué:84.2\%) than in urban areas (Abobo: 78\% and Cocody: $76 \%$ ). It can be noted that for all areas of residence, the practice of intense physical activity at work is inversely related to the degree of education and is also more frequent when the person belongs to the category of the workers than the employees. 


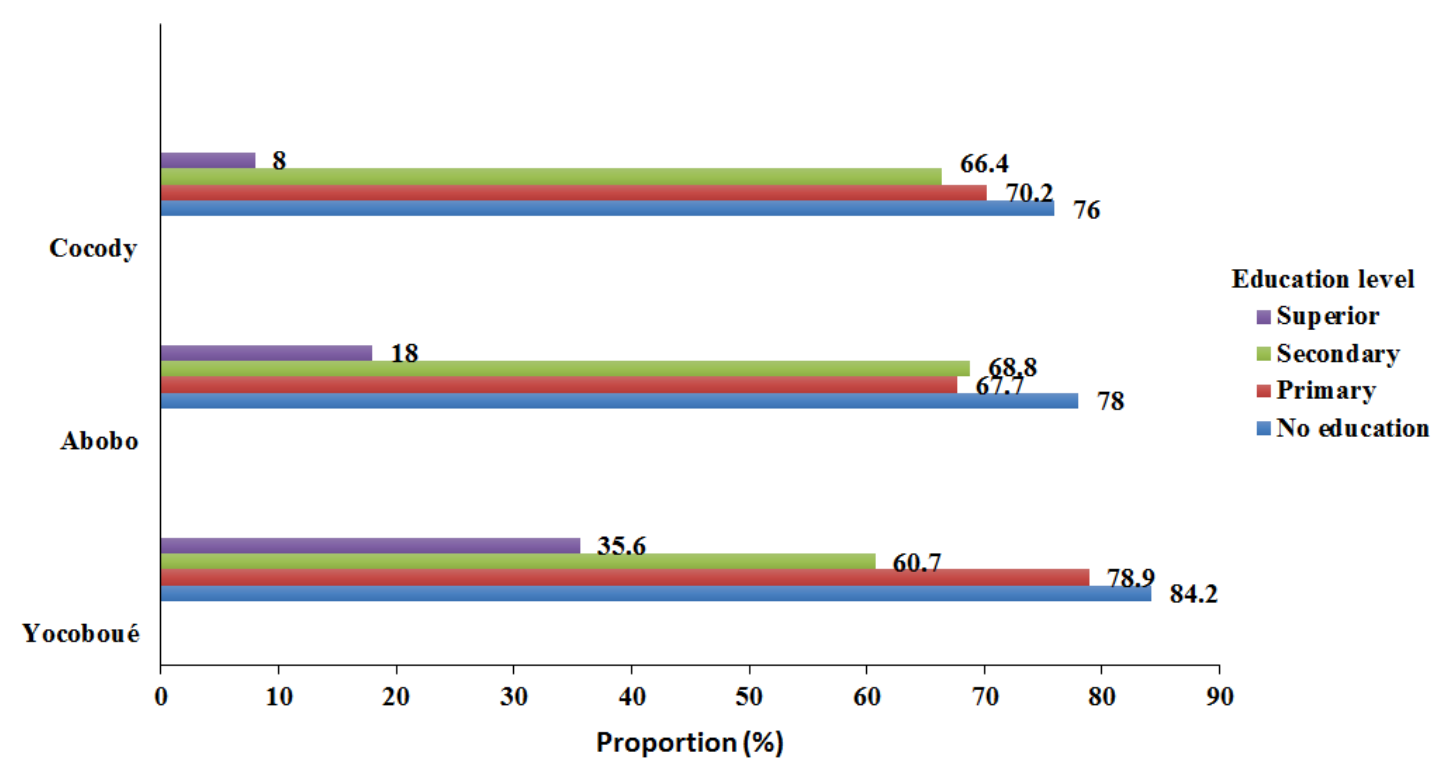

Figure 5. Distribution of the physical activity level according to the study level

\section{Discussion}

\subsection{Practice Contexts according to the Physical Activity Level}

The determination of the physical activity level of the population is a concern in many countries (Maceraet Pratt, 2000) because of the importance of the physical activity in the fight against the chronic diseases, especially in their prevention but also in their management (INSERM, 2008). The Measure of the physical activity level according to the contexts provides a better understanding of the overall physical activity (Berrigan et al., 2006) and (Jacobi et al., 2009).

The consideration of the physical activity in it globality give a better understanding of the fact that the physical activity is practiced in different contexts and can produce effects that are themselves different.

The decrease of the physical activity at work and the displacements from the rural areas to the urban areas may be a reflection of the urbanization with the increase in the mechanization of transport and the sedentarization of the workstations, which is progressively installed (Stamatakis, 2007). This study is consistent with that of Stamatakis (2007) who has showed that the data collected between 1991 and 2004 in England showed a downward trend in physical activity at work.

\subsection{Physical Activity Level according the Age}

The study has also showed that the intense physical activity decreased with age for the entire population. The decrease in intense physical activity with age can be explained by a biological or environmental mechanism. The biological hypothesis is further reinforced by the discovery of dopamine that acts on specific areas of the brain. This hormone is related to the motivation for the locomotion (Sallis and Owen, 1999; U.S., 1996). According to these same authors, other non-biological factors such as environmental (living environment), psychological, social (marital status) and physical (health) variables are also related to the physical activity (Sallis and Owen, 1999; US, 1996). The study corroborates those of the District Control and Prevention Center of Colombia (2004) and of Caspersen et al. (2000) on the decline of age-related physical activity. However, a study conducted on a population of Nigerian civil servants revealed no significant difference between 20 and 64 years of age (Forrest et al., 2001).

\subsection{Physical Activity Level according the Socio-professional Category}

The study has also showed that workers were the most active of all the socio-professional categories; after them we had persons with liberal activities and those with weak activity as office workers. This could be explained by the fact that the workers make enormous efforts in their work. Office workers have activities that require less effort (Oyeyeni et al., 2016). In developed countries people's hobbies tend to include sports and games, however in Côte d'Ivoire as in Nigeria, hobbies tend to be passive with small physical activity (PA) (Oyeyeni et al., 2016).

\subsection{Physical Activity Level according to the Study Level}

The results have showed that the level of education influenced the physical activity level (NAP). This could be 
explained by the fact that low-educated people move regularly or are likely to move by walk and / or by bike than those with a high level of education. People with a high level of education may own a transport vehicle that significantly reduces their level of physical activity (Jurakic et al., 2009).

This study is consistent with those in Mexico, which have showed a higher percentage of adults working in agriculture and fishing, and who were in a higher category of physical activity than those engaged in lower-intensity occupational activities, the latter also having a low or a moderate physical activity level (Salmon et al., 2000).

\subsection{Physical Activity Level according the Sex}

The results have showed that the intense physical activity is more marked in men than in women. This is due to the fact that women generally are engaged in physical activities that range from mild to moderate activity; however, men are engaged in moderate to intense activities (Assahet et al., 2015). Also the African culture have the particularity of making women likely to be employed in work such as: organize the house, do housework, which makes them work less. And generally in this culture women have hobbies that tend to keep them at home, while men tend to indulge in leisure activities that require a physical effort (Walter and Randt, 2011). Our study is in agreement with that of Kiruanda et al. (2016) conducted among peri-urban and rural populations in eastern Uganda where the prevalence of the sedentary in women was higher than that of men. On the other hand, the studies carried out by Oyeyemi et al. (2013) in Nigeria and Kanyoni and philipps (2009) in Rwanda found that women were more active than men.

The urban participants were found to be less active than those in rural areas. This could be due to the fact that the rural communities with their socio-economic status, practiced intense physical activity, such as the agriculture, the main source of income, and a type of active transportation. The urban communities can exercise less intense professional activities or not, or adopt a sedentary lifestyle, due to the fact of their less physically active professional activities and of their motorized mode of transportation (Assah et al., 2015).

Most countries in sub-Saharan Africa, especially those with rapid evolution, are in full phase of demographic and epidemiological development. These development processes have resulted in changes in the social capital of societies, work patterns, and lifestyles that have reduced the physical activity levels (Zewnicki et al., 2003).

\section{Conclusion}

The evaluation of the physical activity level has showed that the physical activity is practiced in different contexts in urban, peri-urban and rural areas. Also, the rural population (Yocoboué) was more active than that of the peri-urban area (Abobo) with moderate physical activity, and that of the urban area (Cocody) with limited physical activity. The low level of physical activity was noticed in the female population. Concerning the socio-occupational category, the office workers had a low physical activity level. For the level of education, the high-educated population had a low level of physical activity. These data suggest that urban residents, women, office workers, and those with low levels of education are targets for the interventions and the physical activity promotion.

\section{References}

Assah, F., Mbanya, J. C., Ekelund, U., Wareham, N., \& Brage, S. (2015). Patterns and correlates of objectively measured free-living physical activity in adults in rural and urban Cameroon. $J$ Epidemiol Community Health, 69(7), 700-707. https://doi.org/10.1136/jech-2014-205154

Barnett, T. A., Gauvin, L., Craig, C. L., \& Katzmarzyk, P. T. (2008). Distinct trajectories of leisure time physical activity and predictors of trajectory class membership: a 22 year cohort study. Interational Journal of Behavioral Nutrition and Physical Activity, 5, 57. https://doi.org/10.1186/1479-5868-5-57

Berrigan, D., Troiano, R. P., McNeel, T., Disogra, C., \& Ballard, B. R. (2006). Active transportation increases adherence to activity recommendations. American Journal of Preventive Medicine, 31(3), 210-216. https://doi.org/10.1016/j.amepre.2006.04.007

Caspersen, C. J., Powell, K. E., \& Christenson, G. M. (1985). Physical activity, execice, and physical fitness: definition and distinctions for health-related research. Public health reports, 100(2), 126-131.

Forrest, K., Bunker, C., Kriska, A., Ukoli, F., Huston, S., \& Markovic, N. (2001) Physical activity and cardiovascular risk factors in a developing population. Medicine and Science in Sports and Exercise, 33, 1598-604. https://doi.org/10.1097/00005768-200109000-00025

Giezendanner, D. (2012). Taille d'un échantillon aléatoire et Marge d'erreur. En ligne: Retrieved from http://icietla-ge.ch/voir/IMG/pdf/7-taille-d_un-echantillon-aleatoire-et-marge-d_erreur-cms-spip.pdf 
Guthold, R., Ono, T., Strong K. L., Chatterji, S., \& Morabia, A. (2008). Worldwide variability in physical inactivity a 51-country survey. Am J Prev Med, 34(6), 486-94. https://doi.org/10.1016/j.amepre.2008.02.013

Haut Comité de la Santé Publique (HCSP). Pour une politique nutritionnelle de santé publique en France. Enjeux et propositions. Rennes: Edition ENSP; 2000.

Institut national de la santé et de la recherche médicale (INSERM). Activité physique: contextes et effets sur la santé. Expertise collective. Paris: INSERM, coll.2008.

Jacobi, D., Charles, M. A., \& Tafflet, M. (2009). Relationships of self reported physical activity domains with accelerometry recordings in French adults. European Journal of Epidemiology, 24(4), 171-179. https://doi.org/0.4000/cybergeo.26711

Kanyoni, M., \& Phillips, J. S. (2009). Factors associated with physical activity levels among older adults in selected institutions in Rwanda. J Commun H Sci., 4(1), 8-14.

Kirunda, B. E., Wamani, H., Fadnes L. T., Broeck J. V. D., \& Thorkild, T. (2016). Objectively Assessed Physical Activity and Associated Factors Among Adults in Peri-Urban and Rural Eastern Uganda: A Population-based Study. Journal of Physical Activity and Health, 13, 1243-1254. https://doi.org/10.1123/jpah.2016-0025

Kohl, Hr., Craig, C., \& Lambert E. (2012). The pandemic of physical inactivity: global action for public health. Lancet, 380(9838), 294-305. https://doi.org/10.1016/S0140 6736(12)608988

Macera, C. A., \& Pratt, M. (2000). Public health surveillance of physical activity. Research Quarterly for Exercise and Sport, 71, 97-103. https://doi.org/10.1080/02701367.2000.11082792

Organisation Mondiale de la Santé (OMS) (2005). Questionnaire mondial sur la pratique d'activités physiques (GPAQ). Guide pour l'analyse. Retrieved from http://www.who.int/chp/steps/GPAQ_Analysis_Guide_FR.pdf

Oyeyemi, A. L., Oyeyeni, A. Y., Adegoke, B. O., Oyetoke, F. O., Aliyu, H. N., \& Aliyu, S. U. (2011). Cross cultural adaptation of the international physical activity Questionnaire: Reliability and validity of the Hausa version in Nigeria. BMC MedicalResearchMethodology, 11(1). https://doi.org/10.1186/1471-2288-11-156

Pepe, H. D. (2017). Les activités physiques comme moyen de lutte contre les maladies non transmissibles en Côte d'Ivoire, mémoire, Master Projet et développement des territoires, Septembre 2017.

Sallis, J. F., \& Owen, N. (2002). Physical Activity and Behavioral Medicine. Thousand Oaks, CA: Sage, 110-133.

Salmon, J., Owen, N., Bauman, A., Schmitz M, K., Booth, M. (2000). Leisure-time, occupational, and household physical activity among professional, skilled, and less-skilled workers and homemakers. Prev Med., 30(3), 191-9.https://doi.org/10.1006/pmed.1999.0619

Stamatakis, E., Hamer, M., \& Lawlor, D. A. (1995). Physical activity, mortality, and cardiovascular disease: is domestic physical activity beneficial? The Scottish Health Survey 1995, 1998, and 2003.young women. Am J PrevMed, 25, 140-143. https://doi.org/10.1093/aje/kwp042

U. S. DEPARTMENT OF HEALTH AND HUMAN SERVICES (1996). Physical Activity and Health: A Report of the Surgeon General. Atlanta, GA: Centers for Disease Control, pp. 215-217, 234-236.

Walter, C. M., \& Randt, R. D. U. (2011). Socio-cultural barriers to physical activity among black isixhosa speaking professional women in the Nelson Mandela metropolitan municipality. S Afr J Res Sport PhysEduc Recreation, 33(2), 143-5. https://doi.org/10.4314/sajrs.v33i2.69698

WHO (2005). Chronic diseases and Health promotion. STEPwise approch to surveilance young women. Am J Prev Med, 25, 140-143.

Zewnicki, R., Vanden Auweele, Y., \& De Bourdeaudhuij, I. (2003). Addressing over reporting on the International Physical Activity Questionnaire (IPAQ) telephone survey with a population sample. Public Health Nutr, 6(3), 299-305. https://doi.org/10.1079/PHN2002427

\section{Copyrights}

Copyright for this article is retained by the author(s), with first publication rights granted to the journal.

This is an open-access article distributed under the terms and conditions of the Creative Commons Attribution license (http://creativecommons.org/licenses/by/4.0/). 